\title{
A Survey on k-NN Classification over Semantically Secure Encrypted Relational
}

\author{
Mayadevi Kotlapure \\ ME Student, Department of Computer Engineering, Sinhgad Academy of Engineering, Pune, Maharashtra, India
}

\begin{abstract}
Data Mining has wide applications in numerous zones, for example, keeping money, prescription, investigative exploration and among government offices. Order is one of the ordinarily utilized assignments as a part of information mining applications. For as far back as decade, due to the ascent of different protection issues, numerous hypothetical and commonsense answers for the order issue have been proposed under diverse security models. Notwithstanding, with the late fame of distributed computing, clients now have the chance to outsource their information, in encoded structure, and also the information mining assignments to the cloud. Since the information on the cloud is in encoded structure, existing security protecting characterization methods are not appropriate. In this paper, we concentrate on fathoming the characterization issue over encoded information. Specifically, we propose a safe $k$-NN classifier over scrambled information in the cloud. The proposed convention ensures the classification of information, security of client's data inquiry, and shrouds the information access designs. To the best of our learning, our work is the first to add to a safe $k$-NN classifier over scrambled information under the semi-legitimate model. Additionally, we exactly dissect the effectiveness of our proposed convention utilizing a genuine dataset under diverse parameter settings.
\end{abstract}

Keyword: Security, k-NN Classifier, Outsourced Databases, Encryption

\section{Introduction}

Of late, the distributed computing model [11] is changing the scene of the associations' method for working their data particularly in the way they spare get to and process information. As a developing handling model, cloud preparing attracts numerous associations to consider truly concerning cloud potential with respect to its expense proficiency, adaptability, and offload of administration cost. Frequently, associations relegate their computational capacities in change to their data to the cloud. Notwithstanding wonderful advantages that the cloud offers, security and solace issues in the thinking are staying away from organizations to use those advantages. At the point when data is greatly sensitive, the data should be encoded before outsourcing to the cloud. All things considered, when data are secured, paying little respect to the genuine security arrangement, executing any data mining errands transforms into extremely muddled while never unscrambling the data. There are other security stresses, affirmed by the accompanying sample. Sample 1: expect a protection supplier gotten its secured customers database and important information mining errand to a cloud. At the point when a delegate from the organization needs to make sense of the risk phase of a potential new customer, the agent can utilize an arrangement system to make sense of the danger phase of the customer. Starting, the delegate requires producing a points of interest history $\mathrm{q}$ for the customer containing certain private subtle elements of the customer, e.g., FICO assessment, age, marriage status, and so on. At that point this history can be sent to the cloud, and the cloud will assess the class mark for q. In any case, since q contains powerless subtle elements, to secure the client's protection, q ought to be encoded before conveying it to the cloud. The above case uncovers that information mining over encoded data (signified by DMED) on a cloud likewise requires securing a client's history when the history is a piece of an information mining strategy. Moreover, cloud can likewise get supportive and fragile data about the genuine data items by observing the data availability styles regardless of the fact that the data are encoded [12], [13]. Consequently, the protection/security details of the DMED issue on a cloud are triple: (11) solace of the encoded data, (12) solace of a client's question history, and (13) covering data availability designs. Current work on security saving information mining (PPDM) (either bother or ensured multi-party calculation (SMC) focused methodology) can't alter the DMED issue. Irritated data don't have semantic insurance, so data bother strategies can't be connected to secure exceptionally sensitive data. Likewise the irritated data don't produce exceptionally exact data mining results. Secure multi-party calculations focused technique speaks to data are spread and not secured at every taking including gathering. In consideration, numerous propelled computations are led relying upon non-encoded data. As a result, in this paper, we recommended novel routines to effectively resolve the DMED issue assuming that the secured data is contracted to a cloud. Especially, we focus on the class issue considering that it is a standout amongst the most widely recognized information mining undertakings. For the reason that every classification methodology has their own advantages, to be unmistakable, this record concentrates on performing the k-closest neighbor classification strategy over secured data in the cloud preparing air.

\section{Related Work}

It is conceivable to utilize the current mystery sharing systems in SMC, for example, Shamir's plan [3], to build up a PPkNN convention. Be that as it may, our work is not the same as the mystery sharing based arrangement in the accompanying angle. Arrangements in view of the mystery sharing plans require no less than three gatherings though our work require just two gatherings. For instance, the developments taking into account Sharemind [4], a surely understood SMC system which depends on the mystery sharing plan, expect that the quantity of taking an interest 


\section{International Journal of Science and Research (IJSR) \\ ISSN (Online): 2319-7064}

Index Copernicus Value (2013): 6.14 | Impact Factor (2014): 5.611

gatherings is three. Therefore, our work is orthogonal to Sharemind and other mystery sharing based plans.

\subsection{Privacy-Preserving Data Mining (PPDM)}

Agrawal and Srikant [5], Lindell and Pinkas [6] were the first to present the idea of privacy preserving under information mining applications. The current PPDM strategies can extensively be grouped into two classes: (i) information bother and (ii) information appropriation. Agrawal and Srikant [5] proposed the first information annoyance system to construct a choice tree classifier, and numerous different strategies were proposed later (e.g., [7][9]). In any case, as specified prior in Area 1, information irritation methods can't be pertinent for semantically secure encoded information. Too, they don't deliver precise information mining results due to the expansion of factual commotions to the information. On the other hand, Lindell and Pinkas [6] proposed the to start with choice tree classifier under the two-party setting IEEE Transactions on Knowledge and Data Engineering Volume: 27 Year: 2015 expecting the information were circulated between them. From that point forward much work has been distributed utilizing SMC systems (e.g., [5]-[25]).

\subsection{Query Processing over Encrypted Data}

Different procedures identified with question handling over encoded information have been proposed, e.g., [24]-[25].On the other hand, we watch that PPkNN is a more mind boggling issue than the execution of basic kNN questions over scrambled information [22], [23]. For one, the middle of the road k-closest neighbors in the order process, should not be revealed to the cloud or any clients. We underline that the late system in [23] uncovers the k-closest neighbors to the client. Furthermore, regardless of the fact that we know the k-closest neighbors, it is still extremely troublesome to discover the lion's share class name among these neighbors since they are encoded at the primary spot to counteract the cloud from learning delicate data. Third, the current work did not tended to the entrance design issue which is a urgent protection prerequisite from the client's viewpoint.

In our latest work [15], we proposed a novel secure k-closest neighbor question convention over scrambled information that ensures information secrecy, client's question protection, and conceals information access designs. Be that as it may, as specified above, PPkNN is a more intricate issue and it can't be explained straightforwardly utilizing the existing secure k-closest neighbor methods over scrambled information. Subsequently, in this paper, we broaden our past work in [15] and give another arrangement to the PPkNN classifier issue over scrambled information. All the more particularly, this paper is not the same as our preparatory work [15] in the accompanying four angles.

\subsection{Threat Model}

We receive the security definitions in the writing of secure multi-party calculation (SMC) [26], [27], and there are three basic antagonistic models under SMC: semi-fair, clandestine and noxious. In this paper, to create secure and proficient conventions, we accept that gatherings are semi-fair.
Quickly, the accompanying definition catches the properties of a safe convention under the semi-fair model [28], [14].

Definition 1: Let ai be the info of gathering Pi, _i(_) be Pi's execution picture of the convention _ and bi be the yield for gathering Pi figured from _. At that point, _ is secure if _i( _) can be reproduced from ai and bi such that dispersion of the mimicked picture is computationally undefined from $\mathrm{i}(\mathrm{C}$. In the above definition, an execution picture by and large incorporates the info, the yield and the messages imparted amid an execution of a convention. To demonstrate a convention is secure under semi-genuine model,we for the most part need to demonstrate that the execution picture of a convention does not release any data with respect to the private inputs of taking an interest parties [14].

\subsection{Paillier Cryptosystem}

The Paillier cryptosystem is an added substance homomorphic also, probabilistic open key encryption plan whose security depends on the Decisional Composite Residuosity Assumption [11]. Let Epk be the encryption capacity with open key pk given by $(\mathrm{N}, \mathrm{g})$, where

$\mathrm{N}$ is a result of two huge primes of comparable piece length and $\mathrm{g}$ is a generator in $\mathrm{Z} * \mathrm{~N} 2$. Additionally, leave Dsk alone the unscrambling capacity with mystery key sk. For any given two plaintexts $a, b \in Z N$, the Paillier encryption plan shows the accompanying properties:

1) Homomorphic Addition

$\operatorname{Dsk}(\operatorname{Epk}(\mathrm{a}+\mathrm{b}))=\operatorname{Dsk}(\operatorname{Epk}(\mathrm{a}) * \operatorname{Epk}(\mathrm{b}) \bmod N 2)$;

2) Homomorphic Multiplication

$\operatorname{Dsk}(\operatorname{Epk}(a * b))=\operatorname{Dsk}(\operatorname{Epk}(a) b \bmod N 2)$;

3) Semantic Security - The encryption plan is

semantically secure [14], [29]. Quickly, given a set of ciphertexts, a foe can't find any extra data about the plaintext(s). For conciseness, we drop the mod N2 term amid homomorphic operations in whatever remains.

\section{Literature Survey}

In this paper [1], another practical method for remote information storage room with proficient availability example solace and rightness is presented. A storage room client can set up this methodology to issue secured read, composes, and embeds to a possibly inquisitive and unsafe storage room administration office, without uncovering data or openness sorts. The supplier is inadequate to set up any association between consequent gets to, or even to separate between a read and a compose. Besides, the buyer is given solid rightness ensures for its capacities - illicit organization conduct does not go unnoticed. We grew first sensible framework requests of greatness faster than present usage that can perform over different questions every second on 1 Tbyte+ databases with full computational solace and accuracy. In paper [2], a totally homomorphic security arrangement is prescribed - i.e., an arrangement that permits one to survey circuits over secured data without having the capacity to decode. Our cure comes in three activities. Starting, we offer a typical result that, to assemble a security plan that permits appraisal of unessential circuits, it suffices to make a security plan that can survey (marginally upgraded

\section{Volume 4 Issue 11, November 2015}




\section{International Journal of Science and Research (IJSR) \\ ISSN (Online): 2319-7064 \\ Index Copernicus Value (2013): 6.14 | Impact Factor (2014): 5.611}

releases of) its own unscrambling circuit; we contact an arrangement that can evaluate its (increased) decoding circuit boots trappable. Forthcoming, we clarify an open key security arrangement utilizing immaculate cross sections that is just about boots trappable. Grid based cryptosystems for the most part have unscrambling calculations with low circuit multifaceted nature, frequently secured with an internal thing calculation that is in $\mathrm{NC1}$. Additionally, culminate cross sections offer both additive and multiplicative homeomorphisms (modulo an open key impeccable in a polynomial band that is appeared as a grid), as required to survey normal circuits. In this paper [3], they show how to separation information $D$ into $n$ things in a manner that $\mathrm{D}$ is rapidly reproduce capable from any $\mathrm{k}$ things, however even complete points of interest of $\mathrm{k}-1$ things indicates unquestionably no insights about D. This procedure permits the improvement of successful key administration methods for cryptographic systems that can work securely and viably notwithstanding when setbacks harm 50 percent the things and assurance breaks uncover everything except one of the staying things. In paper [4], gathering and taking care of fragile information is a testing work. Truth be told, there is no regular recipe for building the important PC. In this archive, they give a provably ensured and proficient broadly useful estimations framework to address this issue. Our answer-SHAREMIND - is a virtual machine for security saving data handling that relies on upon offer processing procedures.

This is a traditional path for securely dissecting components in a multi-party counts air. The one of a kind of our cure is in the decision of the mystery sharing arrangement and the outline of the convention bundle. We have made numerous reasonable decisions to make vast scale examine taking care of conceivable in preparing. The convention of SHAREMIND is data hypothetically ensured in the legit however inquisitive outline with three taking care of individuals. In spite of the fact that the legit however inquisitive outline does not acknowledge hurtful individuals, despite everything it gives extensively enhanced solace upkeep when contrasted with customary unified databases. In this paper [5], the issue of security saving information mining is tended to. Especially, a circumstance in which two gatherings having private databases wish to run an information mining calculation on the organization of their databases, without uncovering any unnecessary subtle elements. Execution is propelled by the require to both ensured blessed points of interest and permit its utilization for examination or different reasons. The above issue is a particular occurrence of ensured multi-party estimations and in that capacity, can be altered utilizing known general convention. In any case, information mining calculations are regularly confounded and, also, the criticism ordinarily incorporates huge subtle elements sets. The general convention in such a case are of no practical use and consequently more successful systems are required. We focus on the issue of choice tree learning with the prominent ID3 calculation. Our convention is fundamentally more powerful than general options and necessities both not very many units of connection and reasonable information exchange data transfer capacity. In paper [6], a structure for mining affiliation rules from dealings made up of specific items where the data has been randomized to ensure solace of individual dealings. While it is conceivable to restore association rules and ensure solace utilizing an uncomplicated "uniform" randomization, the discovered rules can sadly be used to find solace ruptures. Assess the qualities of security ruptures and prescribe a sort of randomization suppliers that are a great deal more proficient than steady randomization in limiting the breaks. At that point get recipe for an impartial bolster estimator and its distinction, which permit us to restore thing set encourages from randomized datasets, and show how to incorporate these equation into investigation techniques. In conclusion, we implementing so as to exist trial results that affirm the criteria it on genuine datasets. In paper [7], the capacity of databases to orchestrate and cooperate frequently enhances solace issues. Information warehousing alongside information mining, giving information from a few assets under a solitary power, enhances the danger of solace offenses. Security ensuring information mining shows a method for managing this issue, particularly if information mining is done in a way that doesn't uncover data past the result. This paper gives a method to freely preparing $\mathrm{k}-\mathrm{nn}$ class from apportioned assets without uncovering any insights about the assets or their information, other than that uncovered by the last classification result. In paper [8], allotted protection safeguarding information digging routines are urgent for mining a few databases with a least data divulgence. We give a structure along a general model and in addition multi-round calculations for investigation side to side parceled databases utilizing a solace ensuring $\mathrm{k}$ Nearest Neighbor $(\mathrm{kNN})$ classifier.

In this paper [9], the issue of helping multidimensional assortment inquiries on secured data is scrutinized. The issue is inspired by protected information freelancing.

\section{Security Analysis of Privacy Preserving Primitives Under the Semi-Honest Model}

First of all, we emphasize that the outputs in the above mentioned protocols are always in encrypted format, and are known only to $\mathrm{P} 1$. Also, all the intermediate results revealed to $\mathrm{P}_{2}$ are either random or pseudo-random. Since the proposed SMIN protocol (which is used as a sub-routine in $\mathrm{SMIN}_{\mathrm{n}}$ ) is more complex than other protocols mentioned above and due to space limitations, we are motivated to provide its security proof rather than providing proofs for each protocol. Therefore, here we only include a formal security proof for the SMIN protocol based on the standard simulation argument [14].

\section{Conclusion}

To secure client protection, different protection saving arrangement strategies have been proposed over the past decade. The current strategies are not appropriate to outsourced database situations where the IEEE Transactions on Knowledge and Data Engineering Volume: 27 Year: 2015 information dwells in scrambled structure on an outsider server. This paper proposed a novel security safeguarding k$\mathrm{NN}$ grouping convention over scrambled information in the cloud. Our convention ensures the secrecy of the information, client's info inquiry, and conceals the 


\section{International Journal of Science and Research (IJSR) \\ ISSN (Online): 2319-7064}

Index Copernicus Value (2013): 6.14 | Impact Factor (2014): 5.611

information access designs. We likewise assessed the execution of our convention under diverse parameter settings. Since enhancing the productivity of SMINn is an imperative initial step for enhancing the execution of our PPkNN convention, we plan to explore elective what's more, more productive answers for the SMINn issue in our future work. Likewise, we will explore and extend our examination to other grouping calculations.

\section{References}

[1] P. Paillier, Public key cryptosystems based on composite degree residuosity classes," in Eurocrypt, pp. 223-238, 1999.

[2] C. Gentry, Fully homomorphic encryption using ideal lattices," in ACM STOC, pp. 169-178, 2009.

[3] A. Shamir, How to share a secret," Commun. ACM, vol. 22,pp. 612-613, Nov. 1979.

[4] D. Bogdanov, S. Laur, and J. Willemson, Sharemind: A framework for fast privacy-preserving computations," in ESORICS, pp. 192-206, Springer, 2008.

[5] R. Agrawal and R. Srikant, Privacy-preserving data mining,"'in ACM Sigmod Record, vol. 29, pp. 439-450, ACM, 2000.

[6] Y. Lindell and B. Pinkas, Privacy preserving data mining," in Advances in Cryptology (CRYPTO), pp. 36-54, Springer, 2000.

[7] P. Zhang, Y. Tong, S. Tang, and D. Yang, Privacy preserving naive bayes classification," ADMA, pp. 744-752, 2005.

[8] A. Evfimievski, R. Srikant, R. Agrawal, and J. Gehrke, Privacy preserving mining of association rules," Information Systems, vol. 29, no. 4, pp. 343-364, 2004.

[9] R. J. Bayardo and R. Agrawal, Đata privacy through optimal k-anonymization," in IEEE ICDE, pp. 217228,2005

[10] H. Hu, J. Xu, C. Ren, and B. Choi, Processing private queries over untrusted data cloud through privacy homomorphism," in IEEE ICDE, pp. 601-612, 2011.

[11] P. Mell and T. Grance, - The NIST definition of cloud computing (draft), $\|$ NIST Special Publication, vol. 800, p. 145, 2011.

[12] S. De Capitani di Vimercati, S. Foresti, and P. Samarati, - Managing and accessing data in the cloud: Privacy risks and approaches, $\|$ in Proc. 7th Int. Conf. Risk Security Internet Syst., 2012, pp. 1-9.

[13] P. Williams, R. Sion, and B. Carbunar, -Building castles out of mud: Practical access pattern privacy and correctness on untrusted storage, $\|$ in Proc. 15th ACM Conf. Compute. Common. Security, 2008, pp. 139148.

[14] O. Goldreich, The Foundations of Cryptography, vol. 2, ch. Encryption Schemes, pp. 373-470. Cambridge University Press, 2004.

[15] Y. Elmehdwi, B. K. Samanthula, and W. Jiang, - Secre k-nearest neighbor query over encrypted data in outsourced environments," in IEEE ICDE, pp. 664675, 2014.

[16] D. Chaum, C. Cr'epeau, and I. Damgard, Multiparty unconditionally secure protocols," in STOC, pp. 11-19, ACM, 1988.
[17] J. Camenisch and M. Michels, Proving in zeroknowledge that a number is the product of two safe primes," in EURO-CRYPT, pp. 107-122, SpringerVerlag, 1999.

[18] Y. Huang, J. Katz, and D. Evans, -Qid-pro-quotocols:Strengthening semi-honest protocols with dual execution," in IEEE Security and Privacy, pp. 272-284, 2012.

[19] Y. Huang, D. Evans, J. Katz, and L. Malka, -Faster secure twoparty computation using garbled circuits," in Proceedings of the 20th USENIX conference on Security (SEC '11), pp. 35-35, 2011.

[20] B. K. Samanthula, Y. Elmehdwi, and W. Jiang, knearest neighbor classification over semantically secure encrypted relational data." eprint arXiv:1403.5001, 2014.

[21] L. Xiong, S. Chitti, and L. Liu, $K$ nearest neighbor classification across multiple private databases," in CIKM, pp. 840-841, ACM, 2006.

[22] W. K. Wong, D. W.-1. Cheung, B. Kao, and N. Mamoulis,-Secure knn computation on encrypted databases," in ACM SIGMOD, pp. 139-152, 2009.

[23] X. Xiao, F. Li, and B. Yao, Secure nearest neighbor revisited,"in IEEE ICDE, pp. 733-744, 2013.

[24] R. Agrawal, J. Kiernan, R. Srikant, and Y. Xu, - Order preserving encryption for numeric data," in ACM SIGMOD, pp. 563- 574, 2004.

[25] B. Hore, S. Mehrotra, M. Canim, and M. Kantarcioglu, Secure multidimensional range queries over outsourced data," The VLDB Journal, vol. 21, no. 3, pp. 333-358, 2012.

[26] A. C. Yao, Protocols for secure computations," in SFCS,pp. 160-164, IEEE Computer Society, 1982.

[27] O. Goldreich, S. Micali, and A. Wigderson, - $-\mathrm{Hw}$ to play any mental game - a completeness theorem for protocols with honest majority," in STOC, pp. 218 229, ACM, 1987.

[28] O. Goldreich, The Foundations of Cryptography, vol. 2, ch. General Cryptographic Protocols, pp. 599-746. Cambridge University Press, 2004.

[29] S. Goldwasser, S. Micali, and C. Rackoff, The knowledge complexity of interactive proof systems," SIAM Journal of Computing, vol. 18, pp. 186-208, February 1989. 